\title{
Bilobol inhibits the lipopolysaccharide-induced expression and distribution of RhoA in HepG2 human hepatocellular carcinoma cells
}

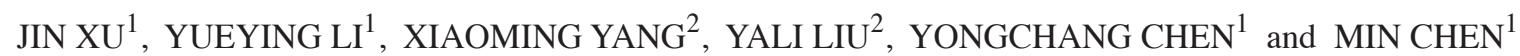 \\ ${ }^{1}$ Department of Anatomy and Physiology, School of Medicine; ${ }^{2}$ Department of Food Science and Engineering, \\ School of Food and Biological Engineering, Jiangsu University, Zhenjiang, Jiangsu 212013, P.R. China
}

Received July 21, 2014; Accepted April 8, 2015

DOI: $10.3892 / 01.2015 .3276$

\begin{abstract}
Recent studies have revealed the localization of RhoA protein in the cell nucleus, in addition to its distribution in the cytosol and cell membrane. The results of previous studies by our group indicated that nuclear RhoA expression is increased, or RhoA is transported into the nucleus, when cells become cancerous or damaged. Furthermore, application of the anticancer agent Taxol appeared to reduce nuclear RhoA localization, indicating an association between the nuclear translocation of RhoA and tumor progression. Bilobol is a traditional Chinese medicine ingredient, however, its anticancer effect has remained unclear. The present study aimed to demonstrate the anticarcinogenic action of bilobol against hepatocellular carcinoma, in order to lay the foundations for subsequent research into the mechanisms underlying its anticancer effects. In the present study, HepG2 cells were treated with lipopolysaccharide (LPS), to induce inflammation, and/or bilobol. By performing an ELISA, it was observed that bilobol was able to suppress the inflammation induced by LPS. In addition, immunofluorescence and western blot analyses indicated that bilobol may reduce the expression of RhoA, suppress translocation of RhoA into the nucleus and inhibit the RhoA/Rho-associated protein kinase signaling pathway. In conclusion, the present study revealed the potential anticancer effects of bilobol.
\end{abstract}

\section{Introduction}

Hepatocellular carcinoma (HCC) is the third leading cause of cancer-related mortalities worldwide. Approximately $80 \%$ of HCCs develop in fibrotic or cirrhotic livers (1). Conventional treatment strategies include surgery, chemo-

Correspondence to: Ms. Yueying Li, Department of Anatomy and Physiology, School of Medicine, Jiangsu University, 301 Xuefu Road, Zhenjiang, Jiangsu 212013, P.R. China

E-mail: yyli@ujs.edu.cn

Key words: bilobol, RhoA, lipopolysaccharide, HepG2 cells therapy and radiotherapy. For the majority of patients with advanced cancer, chemotherapy must be administered following surgery. However, the prognoses of HCC patients, particularly those with high stage or chemoresistant tumors, are still unsatisfactory (2), and the range for radiation therapy is relatively narrow. Other optional treatments include immunotherapy and traditional Chinese medicine. Therefore, the current study investigated the role and mechanisms of bilobol, a traditional Chinese medicine ingredient, in order to understand its antitumor effects and to assess its potential as an antitumor agent or adjuvant agent.

RhoA is a small $\mathrm{G}$ protein, inactive when bound to guanosine diphosphate (GDP) and activated following guanosine triphosphate (GTP) binding. GDP/GTP exchange or GTPase reactions catalyze the conversion between the two forms (3). Rho, a small GTPase, is associated with various cellular functions, including cell adhesion, cell motility and migration, growth control, cell contraction and cytokinesis. Previous studies by our group $(4,5)$ and others $(6,7)$ have indicated that RhoA is located in the nucleus, as well as in the cell membrane and the cytoplasm. In addition, previous investigations conducted by the present research group demonstrated that the nuclear localization of RhoA was affected by numerous factors, including inflammation, damage and certain pharmaceutical agents (5). Furthermore, these previous studies revealed increased nuclear translocation of the RhoA protein when the cell in question is damaged or cancerous.

Bacterial lipopolysaccharide (LPS) is localized to the outer membrane of Gram-negative bacteria, and is capable of activating multiple mammalian cell types and intracellular signaling pathways (8). LPS is a major inflammatory molecule, which induces the production of pro-inflammatory toxins and cytokines, including inducible nitric oxide synthase, cyclooxygenase-2, tumor necrosis factor- $\alpha$ and interleukin-1 (IL-1), IL-6 and IL-8, in various cell types (9). These pro-inflammatory cytokines and bacterial endotoxins induce activation of the Rho GTPase signaling pathway, which mediates pro-inflammatory responses and aggressive carcinogenesis $(10,11)$.

Ginkgo biloba $L$. is a tree native to China, which has been cultivated for over a millennium. In Asia, the Ginkgo biloba 
(Gb) tree has been used medicinally for centuries. Similarly, $\mathrm{Gb}$ leaf extracts are commonly used for a variety of traditional folk remedies in the West (12). Currently, Gb extracts are one of the most commonly administered phytomedicines worldwide, applied for the treatment of neuropsychiatric disorders, asthma, tinnitus, vertigo and cognitive issues $(13,14)$.

The application of ginkgolic acids as anticancer agents has previously been reported $(15,16)$. However, ginkgolic acids are unstable, with bilobol/ginkgo phenol forming easily following heat, acid or base catalysis (17). The cytotoxic evaluation of bilobol as an antitumor agent is in the preliminary stages. Furthermore, an in-depth investigation into the mechanism and effects of bilobol on its targets has commenced (18-20). In consideration of previous studies regarding RhoA by our group, the present study aimed to conduct a broader investigation of the anticancer efficacy of bilobol by analyzing its effect on the distribution of RhoA in HepG2 human hepatocellular carcinoma cells.

\section{Materials and methods}

Cell line. The HepG2 human hepatocellular carcinoma cell line was obtained from the Institution of Cell Biology of the Chinese Academy of Sciences (Shanghai, China).

Reagents. Dulbecco's modified Eagle's medium (DMEM), fetal bovine serum (FBS) and Trypsin-EDTA solution were purchased from Gibco Life Technologies (Grand Island, NY, USA). Fluorescein isothiocyanate (FITC), tetrarhodamine isothiocyanate (TRITC) and horseradish peroxidase (HRP)-conjugated secondary antibodies [goat anti-mouse polyclonal IgG (cat no. 115-005-209) and rabbit anti-goat polyclonal IgG (cat no. 305-005-003)] were purchased from Jackson ImmunoResearch Laboratories, Inc. (West Grove, PA, USA). Furthermore, electrochemiluminescence (ECL) reagents were purchased from GE Healthcare Life Sciences (Chalfont, UK). A Nuclear and Cytoplasmic Extract kit (cat no. KC-435) and antibodies against GAPDH were purchased from Kangchen Biotech, Inc. (Hangzhou, Zhejiang, China). Mouse monoclonal immunoglobulin $\mathrm{G}(\mathrm{IgG})_{1}$ anti-RhoA (cat no. sc-418) and goat polyclonal IgG anti-Rho-associated protein kinase 2 (ROCK2; cat no. sc-1851) antibodies were purchased from Santa Cruz Biotechnology, Inc. (Santa Cruz, CA, USA). In addition, the ELISA kit was obtained from R\&D Systems, Inc. (Minneapolis, MN, USA), LPS was from Sigma-Aldrich (St. Louis, MO, USA) and bilobol (high-performance liquid chromatography purity, >96.5\%) was obtained from the Laboratory of Food and Biological Engineering School, Jiangsu University (Zhenjiang, Jiangsu, China).

Cell culture. HepG2 cells were maintained in DMEM supplemented with $10 \% \mathrm{FBS}$ at $37^{\circ} \mathrm{C}$ in a humidified atmosphere containing $5 \% \mathrm{CO}_{2}$. The medium was changed every two days and the cells were maintained at subconfluence.

Bilobol preparation and cell treatment. Bilobol was dissolved in $0.1 \%$ dimethyl sulfoxide (Bio-Link, York, UK) to obtain a concentration of $15 \mathrm{mg} / \mathrm{ml}$. A total of $2.5 \times 10^{6}$ HepG2 cells were seeded in 6 well plates for use in the Western
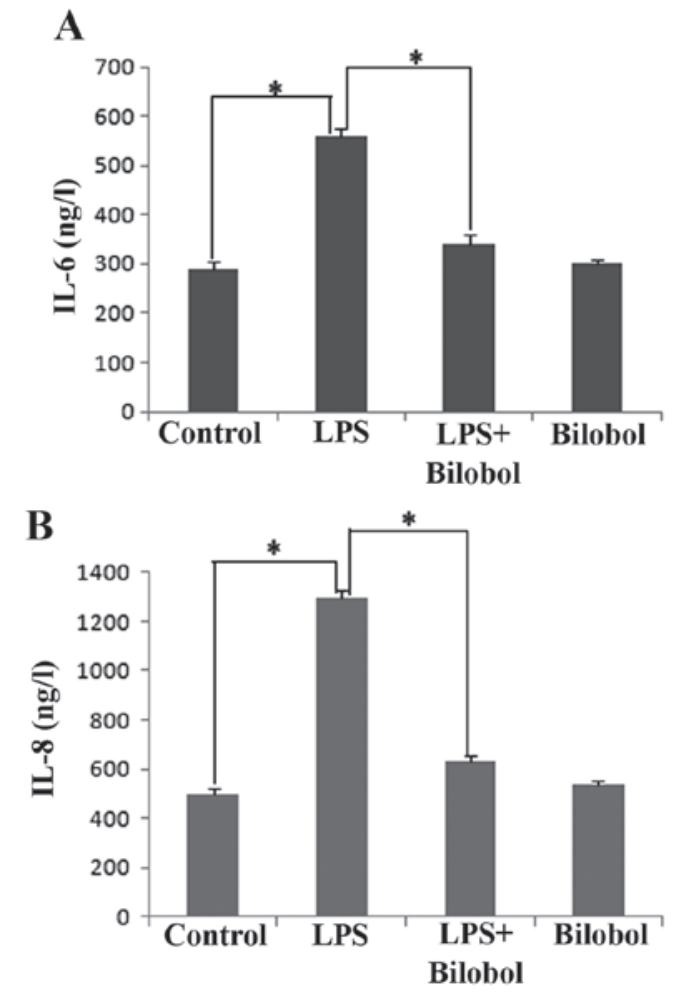

Figure 1. Bilobol rescues the LPS-induced increase in IL-6 and IL-8 expresion. Expression of IL- 6 and IL- 8 induced by LPS or/and bilobol treatment in the HepG2 human hepatocellular carcinoma cell line. HepG2 cells were treated with or without $1 \mu \mathrm{g} / \mathrm{ml}$ LPS and $15 \mu \mathrm{g} / \mathrm{ml}$ bilobol for $12 \mathrm{~h}$. Concentration of (A) IL-6 and (B) IL-8 in the supernatants, determined by ELISA. Data are presented as the mean \pm standard error of the mean $(n=6)$. ${ }^{*} \mathrm{P}<0.05$. LPS, lipopolysaccharide; IL, interleukin.

blot analysis and IL-8/IL-6 expression assays and a total of $5 \times 10^{5}$ HepG 2 cells were seeded in 24 well culture plates for the immunofluorescence assays, and incubated in a humidified atmosphere with $5 \% \mathrm{CO}_{2}$ at $37^{\circ} \mathrm{C}$. When the cells had reached $\sim 80 \%$ confluence, DMEM medium with free serum was added to the wells. Next, $1 \mu \mathrm{M}$ bilobol was added to the 6 and 24-well plates and the cells were cultured for $12 \mathrm{~h}$. Untreated cells were used as the blank control.

Preparation of cytoplasmic and nuclear protein samples. The cytoplasmic and nuclear proteins were extracted from the cultured HepG2 cells according to protocol outlined in the Nuclear and Cytoplasmic Extract kit (Zhejiang Kangchen Biotech Co., Ltd., (Hangzhou, China) manufacturer's instructions. Briefly, the cells were lysed in lysis buffer (50 mM Tris, $150 \mathrm{mM} \mathrm{NaCl}, 1 \mathrm{mM}$ EDTA and 1\% Triton X-100; pH 7.4), collected in an Eppendorf tube (Genetimes Technology, Inc., Shanghai, China), centrifuged at 3,000 x g for $15 \mathrm{sec}$ and suspended in $300 \mu$ l Buffer A. Phenylmethylsulfonyl fluoride (1 $\mu \mathrm{l}$; Shanghai Bogoo Biotechnology Co. Ltd., Shanghai, China), a phosphatase inhibitor (aprotinin; Beijing Leagene Biotech Co., Ltd., Beijing, China), a protease inhibitor mixture, containing leupeptin (Beijing Leagene Biotech Co., Ltd.) and $12 \mu \mathrm{l}$ NP-40 solution were added and the cells were placed on ice for $10 \mathrm{~min}$, then vortexed for $10 \mathrm{sec}$. Next, the cells were placed on ice for $10 \mathrm{~min}$ and centrifuged at $12,000 \mathrm{x} g$ for $15 \mathrm{sec}$. The supernatant, which contained 
A

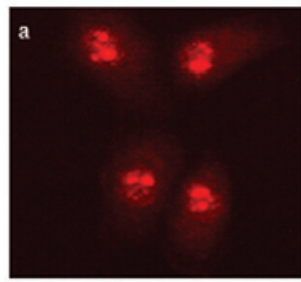

Control

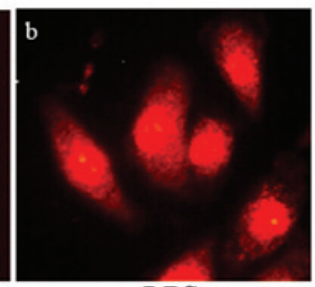

LPS

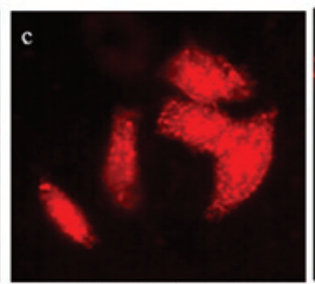

LPS+Bilobol

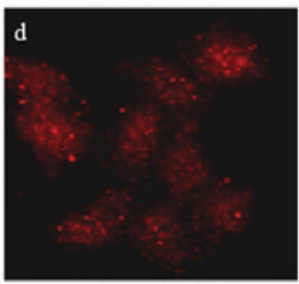

Bilobol

B

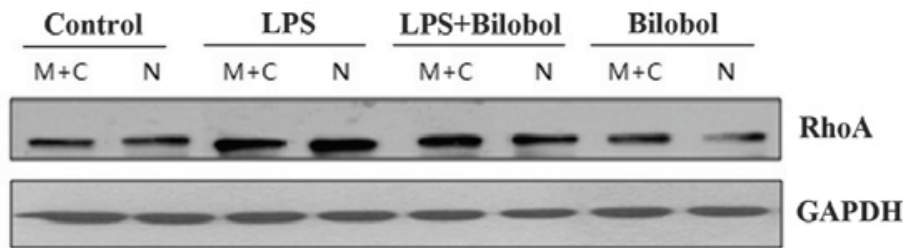

Figure 2. Bilobol inhibits the expression and nuclear localization of RhoA. Expression and distribution of RhoA induced by LPS or/and bilobol treatment in the HepG2 human hepatocellular carcinoma cell line. (A) RhoA was immunofluorescently stained with a specific mouse antibody (Aa) prior to treatment, or following the administration of (Ab) $1 \mu \mathrm{g} / \mathrm{ml}$ LPS, (Ac) LPS plus $15 \mu \mathrm{g} / \mathrm{ml}$ bilobol or (Ad) $15 \mu \mathrm{g} / \mathrm{ml}$ bilobol alone for $12 \mathrm{~h}$ (magnification, x400). (B) Three groups of HepG2 cells were lysed, and the $\mathrm{M}+\mathrm{C}$ and $\mathrm{N}$ fractions were obtained. The expression of RhoA and GAPDH in each fraction was detected by western blotting, and GAPDH expression served as the loading control. LPS, lipopolysaccharide; M+C, membrane and cytosol; N, nucleus.

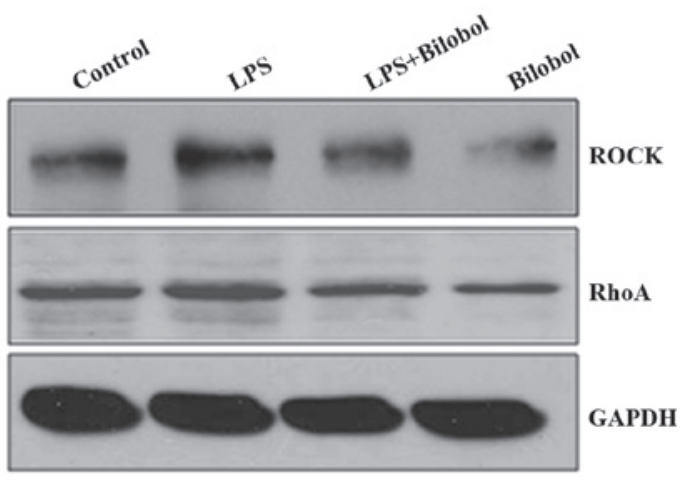

Figure 3. Bilobol inhibits the LPS-induced increased in ROCK2 expression. Protein expression levels of ROCK2 and RhoA induced by LPS or/and bilobol in the HepG2 human hepatocellular carcinoma cell line. Whole cell extracts were immunoprecipitated and protein expression was detected using ROCK2 and RhoA antibodies. GAPDH expression served as the loading control. LPS, lipopolysaccharide; ROCK2, Rho-associated protein kinase 2.

the cytoplasmic protein, was removed and transferred to an Eppendorf tube. The cells were then resuspended in $30 \mu \mathrm{l}$ Buffer B containing protease inhibitors and placed on ice for $30 \mathrm{~min}$. The cells were then centrifuged at $12,000 \mathrm{x} \mathrm{g}$ for $15 \mathrm{sec}$ at $4^{\circ} \mathrm{C}$ and the supernatant was removed. This supernatant contained the nuclear protein. Equal volumes of 2X SDS loading buffer were added to the cytoplasmic and nuclear proteins, respectively. The proteins were then incubated in a Dry Bath Incubator (BG-themoRT; Bay Gene, Inc., Burlingame, CA, USA) at $100^{\circ} \mathrm{C}$ for $5 \mathrm{~min}$.

Immunofluorescence microscopy. HepG2 cells cultured on cover slips were fixed with freshly prepared paraformaldehyde (40 g/l in phosphate-buffered saline; Nantong Jiangtian Chemical Co., Ltd., Nantong, Jiangsu, China) for $30 \mathrm{~min}$. Following penetration with $30 \mathrm{ml} / 1$ Triton X-100 (Beijing Solarbio Science \& Technology Co., Ltd., Beijing, China) and blocking with $30 \mathrm{~g} / \mathrm{l}$ bovine serum albumin (Sigma-Aldrich, St. Louis, MO, USA), the cells were incubated with primary antibodies (dilution, 1:200) at $4^{\circ} \mathrm{C}$ overnight $(\mathrm{o} / \mathrm{n})$. Subsequently, the cells were incubated with TRITC-conjugated polyclonal goat anti-mouse IgG secondary antibodies (dilution, 1:1,000) for $1 \mathrm{~h}$ at room temperature (RT). The cells were washed three times following each incubation. The cellular distribution of the target protein was analyzed under a fluorescence microscope (IX51; Olympus Corporation, Tokyo, Japan).

Western blotting. The sample proteins were run on 10.0 or $12 \%$ SDS polyacrylamide gels. Subsequently, the proteins were transferred onto polyvinyl difluoride (PVDF) membranes (Bio-Rad Laboratories, Inc., Hercules, CA, USA). The PVDF membranes were initially blocked with $5 \%$ milk in Tris-buffered saline and Tween $20(80 \mathrm{~g} / \mathrm{l} \mathrm{NaCl}, 2 \mathrm{~g} / \mathrm{l} \mathrm{KCl}, 30 \mathrm{~g} / \mathrm{l}$ Tris and $0.1 \%$ Tween-20; $\mathrm{pH}$ 7.4) for $1 \mathrm{~h}$ at RT and then incubated with the primary antibodies (dilution, $1: 1,000$ ) at $4^{\circ} \mathrm{Co} / \mathrm{n}$. Following subsequent incubation of the membranes with HRP-conjugated secondary antibodies (dilution, 1:10,000) for $1 \mathrm{~h}$ at RT, ECL reagents were applied, according to the manufacturer' instructions, to reveal the positive bands on the membrane. The bands were detected using a Typhoon 9400 imager (GE Healthcare Life Sciences, Piscataway, NJ, USA).

Determination of IL-8 and IL-6 expression. HepG2 cells were treated with or without LPS $(1 \mu \mathrm{g} / \mathrm{ml})$ for $12 \mathrm{~h}$. The supernatants were then harvested and measured for IL-8 and IL-6 production using an ELISA kit, according to the manufacturer's instructions.

Statistical analysis. All data are expressed as the mean \pm standard error of the mean $(n=6)$. The independent samples t-test was used to compare the expression of IL- 6 and IL-8. The results were analyzed using SPSS software (version 13.0; SPSS, Inc., Chicago, IL, USA). $\mathrm{P}<0.05$ was considered to indicate a statistically significant difference. 


\section{Results}

Bilobol suppresses LPS-induced inflammation. The present study investigated whether LPS was able to mediate the transcriptional activation of the pro-inflammatory genes IL-6 and IL-8, and whether bilobol was able to suppress this LPS-induced inflammatory reaction. HepG2 human hepatocellular carcinoma cells were treated with $1 \mu \mathrm{g} / \mathrm{ml}$ LPS and/or $15 \mu \mathrm{g} / \mathrm{ml}$ bilobol for $12 \mathrm{~h}$, and the concentration of IL- 6 and IL-8 in the supernatants was measured by ELISA. The results indicated that LPS significantly increased the transcriptional activation of IL-6 (Fig. 1A) and IL-8 (Fig. 1B; P<0.05). Furthermore, bilobol was identified to significantly suppress the LPS-induced release of IL-6 (Fig. 1A) and IL-8 (Fig. 1B; $\mathrm{P}<0.05)$. Thus, bilobol exhibited an anti-inflammatory effect.

Bilobol inhibits the expression and nuclear translocation of RhoA. An immunofluorescence assay was performed, in order to determine the level of RhoA expression in the nucleus using an antibody against RhoA. Comparison with control cells revealed that LPS activated RhoA and triggered its translocation to the nucleus in HepG2 human hepatocellular carcinoma cells (Fig. 2Aa and Ab). Furthermore, treatment with bilobol appeared to suppress this effect (Fig. 2Ac and Ad). Thus, the expression of RhoA protein and its localization within the nucleus was increased when the cells were treated with LPS. Bilobol had the opposite effect; it was able to inhibit the two aforementioned phenomena. Subsequent to the immunofluorescent analysis, nuclear extracts were prepared and analyzed by western blotting, using antibodies against RhoA. The western blot analysis results were in agreement with the immunofluorescence data (Fig. 2B), indicating that bilobol inhibits the expression and nuclear translocation of RhoA.

Bilobol inhibits the expression of ROCK2. Western blotting was used to determine the expression of ROCK2 and RhoA, when HepG 2 cells were treated with or without $1 \mu \mathrm{g} / \mathrm{ml}$ LPS and/or $15 \mu \mathrm{g} / \mathrm{ml}$ bilobol for $12 \mathrm{~h}$ (Fig. 3). The expression of ROCK2 was increased following LPS stimulation, however, treatment with bilobol was able to suppress its enhanced expression. ROCK 2 appeared to follow a similar expression pattern to that of RhoA following treatment with LPS and/or bilobol.

\section{Discussion}

RhoA has a molecular mass of $21 \mathrm{kDa}$ and is the most extensively studied member of the Rho GTPase family, which is part of the Ras superfamily of small $G$ proteins. RhoA has been reported to regulate various biological activities, for example gene transcription (21) and tumor progression (22). Furthermore, RhoA activation is typically associated with invasive growth and metastasis, and therefore functions as an oncogene. RhoA is associated with the regulation of numerous biological processes, including stress fiber formation, membrane transport, gene transcription, focal adhesion and tumor progression $(23,24)$.

Ginkgo biloba $L$. is the last surviving member of one of the oldest living seed plant groups, Gymnospermae, and is of medicinal, spiritual and horticultural importance worldwide. $\mathrm{Gb}$ extracts (EGb) are one of the most commonly administered therapeutic agents, and are often prescribed in Europe for use as nootropic agents in old age and dementia (25).

Investigation into the antitumor efficacy of the EGb alkylphenol, ginkgolic acid, has focused on ginkgo acid, specifically. Park et al (16) evaluated the anticancer effects of EGb in estrogen-independent breast cancer. It has been proposed that the chemopreventive effects exhibited by EGb in estrogen receptor-independent breast cancer occur via antiproliferative and apoptosis-inducing activities. Previously, Liu and Zeng (19) identified that HepG2 cells were more sensitive to the cytotoxicity of ginkgolic acid than primary rat hepatocytes. For example, ginkgolic acid appeared to significantly inhibit growth, halt G0/G1 phase progression and decrease B cell lymphoma-2 (Bcl-2)/Bcl-2-associated $\mathrm{X}$ protein expression in HepG2 cells (20), as well as induce the apoptosis of pituitary gland tumor cells by increasing the sensitivity of the cells to radiation (26).

However, alkylphenol acid obtained from EGb is unstable, rapidly forming bilobol/ginkgo phenol under heat, acid or base catalysis. Bilobol is considered to exhibit stronger anticancer properties.

In the present study, LPS appeared to stimulate the nuclear translocation of RhoA protein, while bilobol treatment blocked the subcellular distribution of RhoA under LPS-induced inflammatory conditions. Therefore, in consideration of the results of previous studies by our group, it was proposed that bilobol may exhibit anti-inflammatory and anticancer effects on HepG2 cells. Similarly, standardized EGb 761 and bilobalide treatment have been demonstrated to inhibit inflammation in rats; however, the mechanism of action has remained elusive $(27,28)$. Furthermore, only a small number of studies have been conducted investigating the anti-inflammatory and anticancer effects of bilobol.

The best-known effector of RhoA is ROCK. ROCK appears to be crucial in modulating the force and velocity of actomyosin crossbridging in smooth muscle and non-muscle cells by inhibiting myosin phosphatase-mediated dephosphorylation of the regulatory chain of myosin II (29). In the present study, RhoA/ROCK expression and activity were increased following treatment with LPS, and reduced following the application of bilobol. In addition, bilobol appeared to inhibit the RhoA/ROCK signaling pathway during the anti-inflammatory response and exhibited an anticancer effect. However, the mechanism involved requires further clarification.

In conclusion, the present study identified that the administration of bilobol blocked cancer progression in HepG2 cells. This is in agreement with the results of previous studies by our group, which indicated that the nuclear translocation of RhoA promoted cancer progression. To further assess the antitumor effect of bilobol and develop it into a candidate novel antitumor agent, the antitumor mechanism of bilobol requires additional investigation.

\section{Acknowledgements}

The present study was supported by the National Natural Science Foundation of China (grant no. 81372404), the Specialized Research Fund for Senior Personnel Program of Jiangsu 
University (grant no. 11JDG129), the Postdoctoral Foundation of China (grant no. 2012M521018) and the Postdoctoral Foundation of Jiangsu Province (grant no. 1201025B). These grants were awarded to Ms. Yueying Li.

\section{References}

1. Farazi PA and DePinho RA: Hepatocellular carcinoma pathogenesis: From genes to environment. Nat Rev Cancer 6: 674-687, 2006

2. Forner A, Llovet JM and Bruix J: Hepatocellular carcinoma. Lancet 379: 1245-1255, 2012.

3. Hall A: The cellular functions of small GTP-binding proteins. Science 249: 635-640, 1990.

4. Li Y, Chen Y, Tao Y, Xu J and Chen M: RhoA protein is generally distributed in the nuclei of cancer cells. Oncol Rep 24 1005-1009, 2010

5. Li Y, Chen Y and Xu J: Factors influencing RhoA protein distribution in the nucleus. Mol Med Rep 4: 1115-1119, 2011.

6. Dubash AD, Guilluy C, Srougi MC, Boulter E, Burridge K and García-Mata R: The small GTPase RhoA localizes to the nucleus and is activated by Net1 and DNA damage signals. PLoS One 6 : e17380, 2011.

7. Guilluy C, Dubash AD and García-Mata R: Analysis of RhoA and Rho GEF activity in whole cells and the cell nucleus. Nat Protoc 6: 2050-2060, 2011.

8. Wang Y, Tu Q, Yan W, et al: CXC195 suppresses proliferation and inflammatory response in LPS-induced human hepatocellular carcinoma cells via regulating TLR4-MyD88-TAK1-mediated NF- $\kappa$ B and MAPK pathway. Biochem Biophys Res Commun 456 : 373-379, 2015

9. MacMicking J, Xie QW and Nathan C: Nitric oxide and macrophage function. Annu Rev Immunol 15: 323-350, 1997.

10. Knaus UG: Rho GTPase signaling in inflammation and transformation. Immunol Res 21: 103-109, 2000

11. Wu M, Wu ZF, Rosenthal DT, Rhee EM and Merajver SD: Characterization of the roles of RHOC and RHOA GTPases in invasion, motility and matrix adhesion in inflammatory and aggressive breast cancers. Cancer 116 (Suppl): S2768-S2782, 2010.

12. Hori T, Ridge RW, Tulecke W, Del Tredici P, Tremouillaux-Guiller J and Tobe H (eds): Ginkgo biloba - A Global Treasure. From Biology to Medicine. 1st edition. Springer, Tokyo, 1997.

13. Brondino N, De Silvestri A, Re S, Lanati N, Thiemann P, Verna A, Emanuele E and Politi P: A systematic review and meta-analysis of Ginkgo biloba in neuropsychiatric disorders: From ancient tradition to modern-day medicine. Evid Based Complement Alternat Med 2013: 915691, 2013.

14. von Boetticher A: Ginkgo biloba extract in the treatment of tinnitus: A systematic review. Neuropsychiatr Dis Treat 7: 441-447, 2011.
15. Wu Y, He L, Zhang L, Chen J, Yi Z, Zhang J, Liu M and Pang X: Anacardic acid (6-pentadecylsalicylic acid) inhibits tumor angiogenesis by targeting Src/FAK/Rho GTPases signaling pathway. J Pharmacol Exp Ther 339: 403-411, 2011.

16. Park YJ, Kim MJ, Kim HR, Yi MS, Chung KH and Oh SM Chemopreventive effects of Ginkgo biloba extract in estrogen-negative human breast cancer cells. Arch Pharm Res 36: 102-108, 2013.

17. Yang XM, Wang YF, Li YY, and Ma HL: Thermal stability of ginkgolic acids from Ginkgo biloba and the effects of ginkgol C17:1 on the apoptosis and migration of SMMC7721 cells Fitoterapia 98: 66-76, 2014.

18. Jaggy H, and Koch E: Chemistry and biology of alkylphenols from Ginkgo biloba L. Pharmazie 52: 735-738, 1997.

19. Liu ZH and Zeng S: Cytotoxicity of ginkgolic acid in HepG2 cells and primary rat hepatocytes. Toxicol Lett 187: 131-136, 2009.

20. Zhou C, Li X, Du W, Feng Y, Kong X, Li Y, Xiao L and Zhang P: Antitumor effects of ginkgolic acid in human cancer cell occur via cell cycle arrest and decrease the Bcl-2/Bax ratio to induce apoptosis. Chemotherapy 56: 393-402, 2010.

21. Sotiropoulos A, Gineitis D, Copeland J and Treisman R: Signal-regulated activation of serum response factor is mediated by changes in actin dynamics. Cell 98: 159-169, 1999.

22. Kamai T, Yamanishi T, Shirataki H, Takagi K, Asami H, Ito Y and Yoshida K: Overexpression of RhoA, Racl and Cdc42 GTPases is associated with progression in testicular cancer. Clin Cancer Res 10: 4799-4805, 2004.

23. Price LS and Collard JG: Regulation of the cytoskeleton by Rho-family GTPases: Implications for tumour cell invasion. Semin Cancer Biol 11: 167-173, 2001.

24. Narumiya S: The small GTPase Rho: Cellular functions and signal transduction. J Biochem 120: 215-228, 1996.

25. Kennedy DO and Wightman EL: Herbal extracts and phytochemicals: Plant secondary metabolites and the enhancement of human brain function. Adv Nutr 2: 32-50, 2011.

26. Sukumari-Ramesh S, Singh N, Jensen MA, Dhandapani KM and Vender JR: Anacardic acid induces caspase-independent apoptosis and radiosensitizes pituitary adenoma cells. J Neurosurg 114: 1681-1690, 2011

27. Lee CY, Yang JJ, Lee SS, Chen CJ, Huang YC, Huang KH and Kuan YH: Protective effect of Ginkgo biloba leaves extract, EGb761, on endotoxin-induced acute lung injury via a JNKand Akt-dependent NFאB pathway. J Agric Food Chem 62: 6337-6344, 2014.

28. Goldie M and Dolan S: Bilobalide, a unique constituent of Ginkgo biloba, inhibits inflammatory pain in rats. Behav Pharmacol 24: 298-306, 2013.

29. Wettschureck N and Offermanns S: Rho/Rho-kinase mediated signaling in physiology and pathophysiology. J Mol Med Berl 80: 629-638, 2002. 